\title{
Prevalence of Gingival Hyperplasia Secondary to Amlodipine
}

Veena Kalburgi ${ }^{1}$, Sandhya Raghuwanshi' ${ }^{2}$, Lavanya $\mathrm{N}^{3}$, Madhvi Singh ${ }^{4}$, Somnath Raghuwanshi ${ }^{5}$

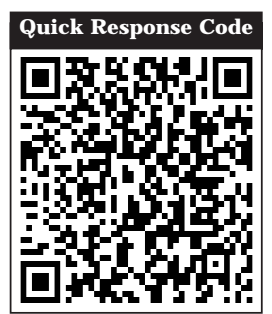

doi : $10.5866 / 2015.7 .10168$

1Professor and HOD

2\&4Post Graduate student

Department of Periodontology,

People's Dental Academy

Bhopal

3Post Graduate student

Department of Periodontology,

PMNM Dental college

Bagalkot

${ }^{5}$ Assistant Professor,

Department of Medicine,

Gandhi Medical college,

Bhopal ( M.P.)

\section{Article Info:}

Received: J uly 10, 2015

Review Completed: August 11, 2015

Accepted: September 14, 2015

Available Online: J uly, 2015 (www.nacd.in)

(C) NAD, 2015 - All rights reserved

\section{Email for correspondence:}

drveenakal burgi@rediffmail.com

\begin{abstract}
:
BACKGROUND: Gingival hyperplasia is one of the side effects associated with certain drugs such as amlodipine, a calcium channel blocker, used as antihypertensive drug. Drug induced gingival enlargement manifests as abnormal growth of the gingiva due to an adverse drug reaction (ADR) in patients treated with anticonvulsants, immunosuppressants, and calcium channel blockers. As gingival enlargement develops, it affects the normal oral hygiene practice and may interfere with masticatory functions. It gradually becomes a source of pain and the condition often leads to disfiguration.
\end{abstract}

AIM: The purpose of the present study is to determine the prevalence and extent of gingival hyperplasia in patients treated with amlodipine for cardiovascular diseases in hypertension and angina.

MATERIALS AND METHODS: A cross-sectional study is undertaken in cardiac patients treated with amlodipine. Information regarding medical history, duration and dosage of medication are recorded and analyzed by clinical parameters such as plaque index and gingival enlargement indices.

RESULTS: In this study, prevalence of gingival hyperplasia was found to be $10 \%$ and association of amlodipine induce gingival hyperplasia with age, sex, duration of medication and plaqueindex were found to be statistically non significant with $p$ value $>0.05$.

CONCLUSION: Gingival hyperplasia could occur with amlodipine even at a small dose $(5 \mathrm{mg})$. Physicians and dentists should beaware of the etiologic medications that can induce gingival hyperplasia.

Key words: Gingival hyperplasia, Amlodipine, Adverse drug reaction.

\section{INTRODUCTION}

Drug-induced gingival enlargement was first reported in1939 by Kimball with chronic usage of the antiepileptic drug phenytoin. ${ }^{1}$ Currently, more than 20 prescription medications are associated with gingival enlargement. ${ }^{2}$ Drug induced gingival overgrowth may be due to hyperplasia of gingival epithelium or of submucosal connective tissue or of both, the major drugs that causes gingival hyperplasia are phenytoin (anti-convulsant), cyclosporine (immunosuppressant) and nifedipine and amlodipine (calcium channel blockers). They interact with epithelial keratinocytes, fibroblasts and collagen, can lead to an overgrowth of gingival tissue in susceptible individuals. ${ }^{3}$ 
Gingival hyperplasia is considered to be a serious adverse effect due to its unaesthetic appearance and also due to its various complications like gingival bleeding, pain, teeth displacement and periodontal disease.

Amlodipine is a new dihydropyridine calcium channel blocker that is used in the management of both hypertension and angina. Ellis et al. first reported gingival sequestration of amlodipine and amlodipine-induced gingival overgrowth.

Amlodipine is given in the dose of $5-10 \mathrm{mg}$ once daily as it has a long duration of action. It is $93 \%$ $98 \%$ protein bound with a volume of distribution of $21 \mathrm{~L} / \mathrm{Kg}$. it is metabolized in liver to inactive metabolite and is mainly excreted via urine.

Amlodipine has main action on vascular smooth muscles It bind to the á-1subunit of L-type calcium channels preferably on the vascular smooth muscles, block calcium entry and reverse vasospasm.

\section{Pathogenesis:}

The pathogenesis of gingival overgrowth is uncertain and treatment is still largely limited to the maintenance of an improved level of oral hygiene and surgical removal of the overgrowth tissues. A number of factors affect the relationship between drug and gingival overgrowth.

\section{Role of fibroblasts}

It has also been proposed that susceptibility or resistance to pharmacologically induced gingival overgrowth may be governed by the existence of differential proportions of fibroblast subsets in each individual which exhibit a fibrogenic response to these medications. In support of this hypothesis, it has been shown that functional heterogeneity exists in gingival fibroblasts in response to various stimuli.

\section{Role of inflammatory cytokines:}

A synergistic enhancement of collagenous protein synthesis by human gingival fibroblasts was found when these cells were simultaneously exposed to nifedipine and interleukin1b (ILIb), a proinflammatory cytokine that is elevated in inflamed gingival tissues. In addition to IL Ib, IL 6 may play a role in the fibrogenic responses of the gingival to these medications. I 6 appears to target connective tissue cells such as fibroblasts both by enhancing proliferation and by exerting a positive regulation on collagen and glycosaminoglycans synthesis. ${ }^{21}$

\section{Role of matrix metalloproteinase:}

Because most types of pharmacological agents implicated in gingival overgrowth have negative effects on calcium ion influx across cell membranes, it was postulated that such agents may interfere with the synthesis and function of collagenases.

Kantarci et al demonstrated that there are significantly higher numbers of basement membrane discontinuities in overgrowth tissues, sometimes containing epitheliallike cells. ${ }^{17}$ Disrupted basal membrane structure in gingival overgrowth tissues is accompanied by a discontinuous collagen type IV expression pattern and decreased laminin5. These findings provided a new additional support for the hypothesis that epithelial plasticity and epithelial to mesenchymal transition promote gingival overgrowth, resulting in compromised basal membrane structure and increased interactions between epithelial and connective tissue layers that contribute to fibrotic pathology. ${ }^{19,20}$

Recently, Subramani et al observed that mast cells participate in many inflammatory oral diseases, particularly those associated with fibrosis. ${ }^{21}$ They possess very diverse roles ranging from proinflammatory to immunomodulatory. Upon their activation, they promote the local renin angi otensin system generation consequently able to stimulate endothelin and other profibrotic mediators. Cyclosporin can modulate local expression of renin angiotensin system components such as angiotensinogen, angiotensin II and its receptors in gingival tissues, and gingival fibroblast cells. ${ }^{21,} 22$

\section{Materials and Methods:}

Potential subjects for this study were identified randomly from the Department of Periodontology, of People's Dental Academy, Bhanpur, Bhopal. Sixty qualified individuals volunteered to participate in the study. After obtaining written consent, an interview and oral examination were performed on each subject. The following parameters were recorded: age; sex; Loe and Sillness Plaque Index, amlodipine dosage, duration and gingival hyperplasia, using the grading system described by Angelopoulos and Goaz 1972 (Grade 0: No signs of gingival enlargement, Grade 1: Enlargement confined to interdental papilla, Grade 2: Enlargement involves papilla and marginal gingival, 
Grade 3: Enlargement involves three quarters or more of the crown) (Figure 1, 2 and 3).

Selection criteria included: 1) medication with amlodipine, $5 \mathrm{mg}$ per day, for at least 6 months; 2) at least 10 natural teeth; 3) no other medication known to be associated with gingival hyperplasia; 4) no antibiotic premedication required for dental treatment; and 5) no periodontal treatment within the previous 6 months.

\section{Data Analysis:}

Chi-square test was used to determine any relationship of gender, drug dosage and duration with gingival hyperplasia, and independent t-test analyses were conducted to assess the role of age, plaque and gingival indices, by comparing groups with and without gingival hyperplasia. Karl-Pearson correlation coefficient between probing depth and gingival index score was also determined.

\section{Results:}

Overall results are presented in Table 1. Thirty eight males and twenty two females, aged 31 to 79 years, were examined. All subjects had been taking amlodipine for at least 6 months (mean duration: 28 months), and all reported good compliance. Average Plaque indices 31\%.

Mild gingival hyperplasia (score of 2) was detected in six subjects-a prevalence of $10 \%$. No correlations were found between gingival hyperplasia and age, sex, duration of medication, or plaque index. While no statistically significant relationship was found in this study between plaque and hyperplasia. The most common dosage of amlodipine is $5 \mathrm{mg}$ per day and was chosen for this study to facilitate obtaining a large sample size.

In summary, 60 dentate patients (38 males, 22 females), 31 to 79 years of age, who had been taking amlodipine (5mg per day for at least 6 months), were examined for gingival hyperplasia (Graph 1). Moderate hyperplasia was detected in six patientsa prevalence rate of $10 \%$, and association of amlodipine induce gingival hyperplasia with age, sex , duration of medication and plaque index were found to be statistically non-significant with $p$ value>0.05.

\section{DISCUSSION}

Amlodipine is a 3rd generation dihydropyridine calcium antagonist which is structurally similar to nifedipine but pharmacodynamically comparable to it. In patients with hypertensive heart disease the prevalence of gingival overgrowth associated with amlodipine is lower than that associated with other calcium channel blocking agents including nifedipine. ${ }^{6}$ Drug-induced gingival overgrowth usually occurs within the first 3 months of starting drug therapy at a dose of 10mg/day and begins as an enlargement of the interdental papilla. Although few cases of amlodipine induced hyperplasia have been reported, the present case is interesting as it occurred with a low dose of amlodipine (5mg) and appeared on administration for 6 months.

Seymour et al. ${ }^{8}$ gave a review on the pathogenesis of drug-induced gingival overgrowth in which they considered it as amultifactorial model, involving an interaction of several factors, which expands on the interaction between drug and metabolite with the gingival fibroblasts. Predisposing factors for these changes are age, genetic predisposition, pharmacokinetic variables, drug-induced alterations in gingival connective tissue homeostasis, histopathology, ultrastructural factors and inflammatory changes, and druginduced action on growth factors.

The underlying mechanism behind druginduced gingival hyperplasia involves inflammatory and noninflammatory pathways. The proposed noninflammatory mechanisms include defective collagenase activity due to decreased uptake of folic acid, blockage of aldosterone synthesis in adrenal cortex, and consequent feedback increase in adrenocorticotropic hormone level and upregulation of keratinocyte growth factor. Alternatively, inflammation may develop as a result of direct toxic effects of concentrated drug in crevicular gingival fluid and/or bacterial plagues. This inflammation could lead to theupregulation of several cytokine factors such as transforming growth factor-51. 9, 11

Many studies have been conducted which showed that amlodipine cannot induce gingival hyperplasia at $5 \mathrm{mg}$ once daily dose even if taken for more than 6 months. It can be caused only at a dose of $10 \mathrm{mg} /$ day. 6,8 The present case is unique in that even $5 \mathrm{mg} /$ day dose of amlodipine caused gingival hyperplasia after 6 months of use.

The mechanism through which these drugs induce gingival enlargement is still poorly understood. It has been found that phenytoin and calcium channel blockers inhibit the intracellular 


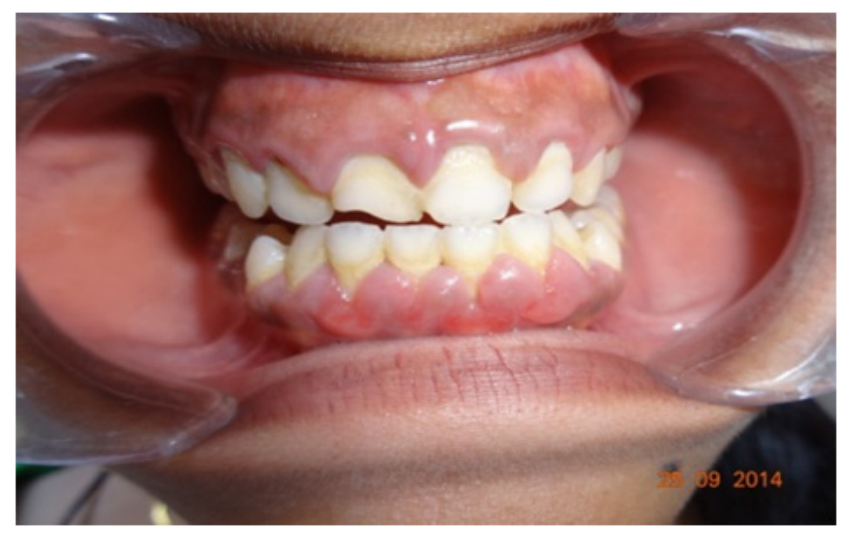

Figure 1: Amlodipine-induced Grade I enlargement

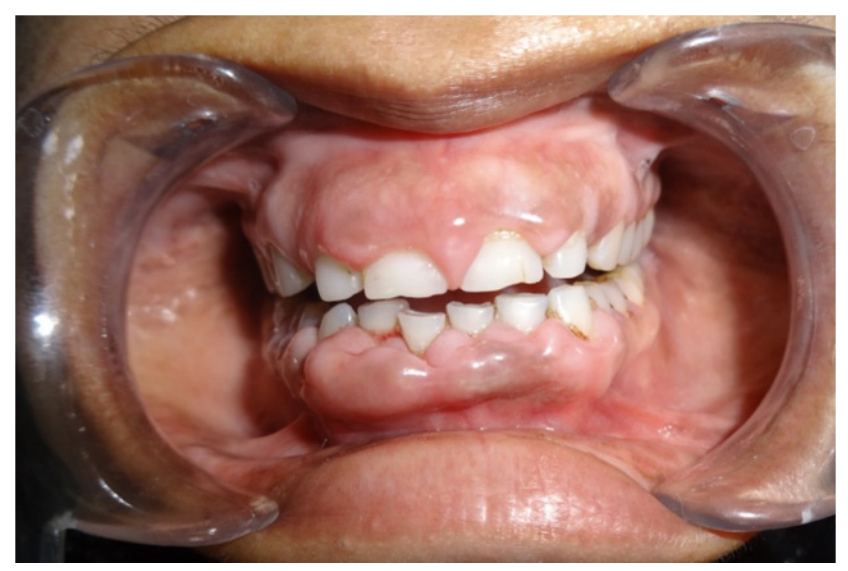

Figure 3: Amlodipine-induced Grade III enlargement

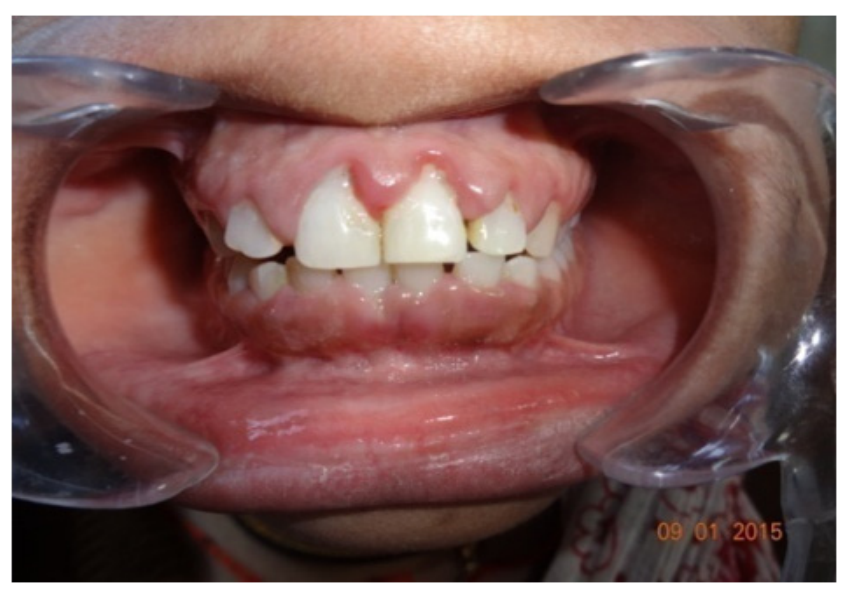

Figure 2: Amlodipine-induced Grade II enlargement

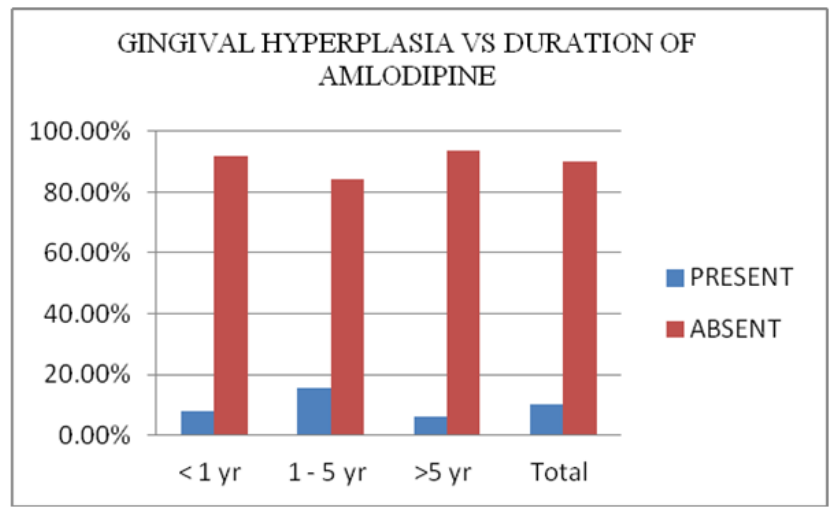

Graph 1: Gingival hyperplasia and duration of amlodipine.

Table 1- sex, age range, plaque index, and Prevalence of Gingival Hyperplasia

\begin{tabular}{llcccc} 
subject & $\mathbf{n}$ & Age $(\mathbf{y r})$ & Plaque Index & Gingival Hyperplasia & $\%$ \\
Male & 38 & $31-75$ & $42 \%$ & 4 & 10.52 \\
\hline female & 22 & $40-79$ & $20 \%$ & 2 & 9.09 \\
\hline Total & 60 & $31-79$ & $31 \%$ & 6 & 10.00 \\
\hline
\end{tabular}

Ca2+ uptake thereby stimulating gingival fibroblasts. Not all the patients receiving the same drug developgingival enlargement. Possible reason can be that individuals with gingival enlargement have fibroblasts with an abnormal susceptibility to the drug. It has also been proposed that the susceptibility to pharmacologically induced gingival enlargement may be governed by existence of differential proportions of fibroblast subset in each individual which exhibit a fibrogenic response to these medications. It has also been shown that the functional heterogenicity exists in gingival fibroblasts in response to various stimuli. ${ }^{12}$
A synergestic enhancement of collagenous protein synthesis by human gingival fibroblasts is found when these cells are exposed simultaneously to calcium channel blockers and elevated levels of interleukin-15 (a proinflammatory cytokine) in inflamed gingival tissues. Interleukin-6 also plays a role in fibrogenic responses of gingiva to these medications. I nterleukin- 6 targets fibroblasts which trigger the proliferation of fibroblasts and exert the positive regulation on collagen and glycosaminoglycans synthesis. So this cytokine has been proposed to play a pathogenic role in fibrotic gingival enlargement. ${ }^{8}$ 
Clinical relevant doses of cyclosporins trigger gingival fibroblasts to exhibit significant reduced levels of matrix metalloproteinases- 1 and -3 secretions which lead to accumulation of extracellular matrix components. ${ }^{13}$ There is a strong correlation between the production of inactive collagenase and responding fibroblasts. Because of reduced folic acid uptake, there is limited production of activator protein which converts inactive collagenase to active collagenase. Limited amount of collagenase becomes available. ${ }^{8}$

Treatment consists of stopping the offending drug if possible with the patient's physician consent and providing the supplements of folic acid and ascorbic acid. Reduction in the size of the gingival overgrowth has been reported within a week of drug withdrawal and may lead to full resolution. ${ }^{14}$

Patients benefit from effective oral hygiene measures, professional tooth cleaning, scaling, and root planning. ${ }^{15}$ If gingival enlargement persists after careful consideration of the previously mentioned approaches, these cases need to be treated by surgery, either by gingivectomy or flap surgery.

\section{Conclusion}

We conclude that the gingival hyperplasia could occur with amlodipine even at a small dose $(5 \mathrm{mg})$. Physicians and dentists should be aware of the etiologic medications that can induce gingival hyperplasia and be able to identify changes in the oral cavity in such patients and to prevent, diagnose, and successfully manage them. It can be treated locally and systemically with combined effort of medical and dental physician. So, cooperative teamwork between the patients, physician, and the dental health care professional is mandatory to minimizeand successfully treat such unwanted side effects of drugs.

\section{REFERENCES}

1. Pradhan S, Mishra P. Gingival enlargement in antihypertensive medication. J Nep Med Assoc 2009; 48(174):149-152.

2. Rees D, Levine RA. Systemic drugs as a risk factor periodontal disease initiation and progression. Comp Cont Edu Dent 1995; 16(1):20-42.

3. Shan Sasidharan et al. Int. J. Bioassays 2015: 4(01):36783679.

4. Michael J orgensen G. Prevalence of amlodipine -related Gingival hyperplasia. J Periodont 1997; 68:676-678.
5. Drug associated gingival enlargement. J Periodont 2004; 75(10):1424-1431.

6. Seymour RA, Thomson J S, Monkman S, I dle J R. Amlodipine -induced gingival overgrowth. J Clin Periodont 1994; 21:281283.

7. Ellis J S, Seymour RA, Steel e J G, Robertson P, Butler TJ Thomason J M. Prevalence of gingival overgrowth induced by calcium channel blockers: a community-based study. J Periodont 1999; 70(1):63-67.

8. J orgensen MG. Prevalence of amlodipine-related gingival hyperplasia. J Periodont 1997; 68(7):676-678.

9. Triveni MG, Rudrakshi C, Mehta DS. Amlodipine induced gingival overgrowth. J Ind Soc Periodont 2009; 13(3):160163.

10. Seymour RA, Thomason J M, Ellis J S. The pathogenesis of drug-induced gingival overgrowth. J Clin Periodont 1996; 23(3):165-175.

11. Nyska A, Shemesh M, Tal H, Dayan D. Gingival hyperplasia induced by calcium channel Sblockers: mode of action. Medical Hypotheses 1994; 43(2):115-118.

12. Marshall RI, Bartold PM. A clinical review of druginduced gingival overgrowths. Aust Dent J 1999; 44(4):219-232.

13. Lafzi A, Farahani RMZ, Shoja MM. Amlodipine induced gingival hyperplasia. Medicina Oral, Patologia Oral Cirugia Bucal 2006; 11(6):480-482.

14. Seymour RA. Calcium channel blockers and gingival overgrowth. Brit Dent J 1991; 170(10):376-379.

15. Sonmez S, Cavdar C, Gunduz C et al. Do MMP-1 levels of gingival fibroblasts have a role in the gingival overgrowth of cyclosporine-treated patients. Transplantation Proceedings 2008; 40(1):181-183.

16. Raman PG, Mishra VN, Singh D. Nifedipine induced gingival hyperplasia. TheJ Assoc Phys Ind 1988; 36(3):231233.

17. Mavrogiannis M, Ellis J S, Thomason J M, Seymour RA. The management of drug-induced gingival overgrowth. J Clin Periodont 2006; 33(6):434-439.

18. Dongari Baqtzoglou A. Research, Science and therapy committee, american academy of periodontology. Drugassociated gingival enlargement. J Periodontol 2004; 75:142431.

19. Kantarci A, Nseir Z, Kim YS, Sume SS, Trackman PC. Loss of basement membrane integrity in human gingival overgrowth. J Dent Res 2011; 90:88793.

20. Sume SS, Kantarci A, Lee A, Hasturk H, Trackman PC. Epithelial to mesenchymal transition in gingival overgrowth. Am J Pathol 2010; 177:20818.

21. Subramani T, Rathnavelu V, Yeap SK, Alitheen NB. Influence of mast cells in druginduced gingival overgrowth. Mediators Inflamm 2013; 275.

22. Subramani T, Senthilkumar K, Periasamy S, Rao S. Expression of angiotensin II and its receptors in cyclosporine-induced gingival overgrowth.J Periodontal Res 2013; 48:386-391. 